\title{
In Vitro and In Silico Cytotoxic and Antibacterial Activities of a Diterpene from Cousinia alata Schrenk
}

\author{
Almira Zhanzhaxina $\mathbb{D}^{1,2}$, Yerlan Suleimen ${ }^{10}{ }^{3}$ Ahmed M. Metwaly ${ }^{(D)}{ }^{4}$ \\ Ibrahim H. Eissa $\mathbb{D}^{\mathbb{D}},{ }^{5}$ Eslam B. Elkaeed $\mathbb{D},{ }^{6}$ Raigul Suleimen $\mathbb{D}^{\mathbb{D}},{ }^{1}$ Margarita Ishmuratova $\mathbb{D}^{\mathbb{D}},{ }^{7}$ \\ Kydyrmolla Akatan $\mathbb{D}^{8}$, and Walter Luyten $\mathbb{B D}^{9}$ \\ ${ }^{1}$ L.N. Gumilyov Eurasian National University, Nur-Sultan, Kazakhstan \\ ${ }^{2}$ S. Seifullin Kazakh Agro Technical University, Nur-Sultan, Kazakhstan \\ ${ }^{3}$ Sh. Ualikhanov Kokshetau University, Kokshetau, Kazakhstan \\ ${ }^{4}$ Pharmacognosy and Medicinal Plants Department, Faculty of Pharmacy, Al-Azhar University, Cairo, Egypt \\ ${ }^{5}$ Pharmaceutical Medicinal Chemistry Department, Faculty of Pharmacy, Al-Azhar University, Cairo, Egypt \\ ${ }^{6}$ Department of Pharmaceutical Sciences, College of Pharmacy, AlMaarefa University, Ad Diriyah, Riyadh 13713, Saudi Arabia \\ ${ }^{7}$ E.A. Buketov Karaganda University, Karaganda, Kazakhstan \\ ${ }^{8}$ S. Amanzholov East Kazakhstan State University, Oskemen, Kazakhstan \\ ${ }^{9}$ Department of Biology, Katholieke Universiteit Leuven, Leuven 3000, Belgium
}

Correspondence should be addressed to Yerlan Suleimen; syerlan75@yandex.kz and Ahmed M. Metwaly; ametwaly@azhar.edu.eg

Received 7 February 2021; Revised 18 March 2021; Accepted 3 April 2021; Published 19 April 2021

Academic Editor: Teodorico C. Ramalho

Copyright (C) 2021 Almira Zhanzhaxina et al. This is an open access article distributed under the Creative Commons Attribution License, which permits unrestricted use, distribution, and reproduction in any medium, provided the original work is properly cited.

\begin{abstract}
A biologically guided isolation of secondary metabolites from Cousinia alata Schrenk wild plant growing in Akmola region, Kazakhstan, led to the isolation of the bioactive diterpene grindelic acid (1). Six flavonoids were also isolated and identified as retusine (2), pachipodol (3), jaranol (4), penduletin (5), casticin (6), and 5, 7, 3' -trihydroxy-3, 4' -dimethoxyflavone (7). Penduletin (5) showed moderate cytotoxic activity assay. Grindelic acid exhibited promising cytotoxic activity against the Artemia salina nauplii and antibacterial activity against Staphylococcus aureus, Bacillus cereus, and Salmonella enteritidis. The presence of the essential pharmacophoric features of histone deacetylase (HDAC) inhibitors in the structure of grindelic acid encouraged us to run a molecular docking study against the HDAC enzyme to understand its mechanism of action on a molecular level. Grindelic acid showed a binding mode of interaction similar to that of the cocrystallized ligand and exhibited good binding affinity against HDAC with the binding free energy of $-18.70 \mathrm{kcal} / \mathrm{mol}$. The structures of isolated compounds were determined by MS, 1D, and 2D NMR spectroscopy methods. Compounds (1-7) were isolated for the first time from Cousinia genus.
\end{abstract}

\section{Introduction}

Cancer is a disease that takes place in an organ or tissue of the body because of the uncontrolled growth of abnormal cells invading other parts inside the body and spreads to other tissues [1,2]. According to the WHO, in 2018, with a ratio of one in six deaths, cancer was the second cause of death all over the world [3]. The global increase in antibiotic resistance causes an increase in medical costs and mortality rates, especially with some severe bacterial infections such as pneumonia, tuberculosis, gonorrhea, and salmonellosis [4]. Natural products can be considered as the main source of antimicrobial and anticancer compounds [5]. The activity of natural products either originated from plants [5-7], marines [8,9] or fungi [10-14] is owned to a diversity of secondary metabolites that belong to various chemical classes such as flavonoids [15-17], saponins [6, 18], pyrones [19], isochromenes [20], and alkaloids [21].

Cousinia Cass. is one of the largest genera of the Asteraceae family with approximately $600-700$ species and 
the most diverse species in Central and Southwest Asia [22-24]. About 56 species are distributed throughout Kazakhstan, including Cousinia alata Schrenk., which is a perennial plant, up to $50-90 \mathrm{~cm}$ high; its stems are straight, freely branched, naked, and winged; wings are with spinydentate margin; leaves are thin and simple; corolla is yellow or pink color. During the flowering period from flower baskets, there is a significant release of rubber. This plant grows on the slopes of low hills, in sandy places, and in plains throughout Kazakhstan's territory. General distribution: China, Mongolia, and Central Asia [25-27].

The bioactivity-guided isolation led to the identification of cytotoxic compounds from four different Cousinia species (Cousinia davisiana, Cousinia foliosa, Cousinia ramosissima, and Cousinia stenocephala) [28]. Moreover, the methanolic extracts of C. davisiana Hub.-Mor., C. foliosa Boiss., C. ramosissima DC., and C. stenocephala exhibited good cytotoxic activity on A549, Beas-2b, and Colo205 cell lines [29]. Furthermore, the cytotoxic effect of C. verbascifolia Bunge against OVCAR-3 and HT-29 cancer cells was reported [30]. Also, cytotoxicity of C. alata Schrenk essential oil was determined using the brine shrimp assay [27]. On the other hand, the antimicrobial properties of different Cousinia species have been proved $[31,32]$. Additionally, some reports discussed the antioxidant effect of Cousinia species [29, 33]. A diverse of secondary metabolites have been isolated from the genus of Cousinia including polysaccharides and their monosaccharide [34, 35] and sesquiterpene lactones [36, 37], in addition to flavonoids, tannins, glycosides, terpenoids, phenols, and saponins [22, 31].

Histone deacetylases (HDAC) are a group of enzymes that are responsible for the deacetylation from the $\mathrm{N}$-acetyl lysine amino acid on the histone protein; this step (deacetylation) is very essential in the process of DNA expression because it allows the histones to wrap the DNA more effectively [38]. HDAC inhibitor is any molecule that increases the acetylation of lysine residues on histone and nonhistone proteins through the inhibition of HDAC enzyme activity. Consequently, HDAC inhibitors could be an excellent choice to discover new anticancer agents [39]. Moreover, the presence of HDAC homologs in bacteria in the form of acetoin utilization proteins (AcuC) has been reported [40]. Also, the use of a histone deacetylase inhibitor in infected mice with $M$. tuberculosis inhibited bacterial growth, accelerated immune cell recruitment, induced proinflammatory cytokine expression, and suppressed IL-10 expression [41]. Another model of infected mice with E. coli treated with valproic acid (HDAC inhibitor) caused a noticeable decrease in bacterial load exaggeration and cytokine expression [42]. Recently, Grabiec et al reported the antibacterial effects of several HDAC inhibitors through different mechanisms [43].

This study aims to isolate and identify the secondary metabolites which are responsible for the cytotoxic and antibacterial effects of Cousinia alata Schrenk extracts. Additionally, molecular docking studies have been preceded to have a better idea about the mechanism of action of the active compound on a molecular level.

\section{Experimental Part}

2.1. Extraction, Isolation, and Purification. The aerial parts of C. alata Schrenk (Asteraceae) were collected during the flowering period in the vicinity of Zhaltyr village (Almaty-Ekaterinburg Highway) of Astrakhan District, Akmola region, Republic of Kazakhstan. A voucher specimen (no. 2002.07.23.02.04) was deposited in the Herbarium of the Biology and Geography Faculty, E.A. Buketov Karaganda University.

The air-dried flowers and leaves of C. alata $(500 \mathrm{~g})$ were extracted with chloroform in the water bath at $65^{\circ} \mathrm{C}$ for one hour. After filtration, the filtrate was evaporated under reduced pressure at $40^{\circ} \mathrm{C}$. This procedure was repeated three times and the concentrated extracts were treated with waterethanol $(1: 2)$ solution to remove ballast substances. After water-ethanol treatment, the obtained extract was subjected to silica-gel column chromatography. Column chromatography runs were performed using silica gel $(63-200 \mu \mathrm{m}$, $735 \mathrm{~g}$ ) and a mobile phase of petroleum ether/ethyl acetate with a manner of increasing polarity, which afforded 120 fractions (each $250 \mathrm{ml}$ ).

All fractions were subjected to TLC plates (silica gel 60 $\left.\mathrm{F}_{254}\right)$. Fractions $29-34 \quad 85: 15 \%$, petroleum ether: ethyl acetate) were combined and subjected to TLC $\left(\mathrm{CHCl}_{3}\right.$ : $\mathrm{MeOH}, 98 \%: 2 \%$ ), which showed that it is a mixture of two compounds $(217 \mathrm{mg})$. Then, this mixture was purified by prep. TLC (silica gel $60 \mathrm{~F}_{254} 2 \mathrm{~mm}, 20^{*} 20 \mathrm{~cm}, \mathrm{CH}_{2} \mathrm{Cl}_{2^{-}}$ $\mathrm{MeOH}, 99 \%: 1 \%$ ) to give $31 \mathrm{mg}$ of compound (2) and identified by NMR spectrometry as 5-hydroxy-3, 3', 4', 7tetramethoxyflavone (retusine). Fractions 39-43 (75:25) contained a mixture of compounds (3) and (4), which were separated on silica gel column, the solvents gradient $\mathrm{CHCl}_{3}$ : $\mathrm{MeOH}(40: 1)$, and by NMR results, they were identified as pachipodol and jaranol, respectively. The group of fractions $44-48,49-57$, and $63-70$ were combined and then purified using recrystallization/washing with a mixture of solvents (petroleum ether: ethyl acetate). As a result, this led to the identification of penduletin (5), casticin (6), and 5, 7, $3^{\prime}$ trihydroxy-3, 4' -dimethoxyflavone (7), respectively.

Regarding isolation of grindelic acid (1), $200 \mathrm{~g}$ of the aerial parts of $C$. alata were powdered and extracted with methanol, followed by evaporation under reduced pressure on a rotary evaporator. The crude methanol extract was further fractionated by liquid-liquid extraction with hexane and then dichloromethane. The fractions were then concentrated again using a rotary evaporator to give hexane $(12,3 \mathrm{~g})$, dichloromethane $(25,8 \mathrm{~g})$, and methanol extracts. A sample was taken from each extract for preliminary screening of antibacterial activity. Thus, the hexane extract showed antimicrobial activity, which was further subjected to bioassay-guided isolation of antimicrobial compounds. The dried active extract (hexane) was loaded on a silica gel column $(60 \mathrm{~cm}$ height and $5.5 \mathrm{~cm}$ diameter), which was eluted with a step gradient increasing polarity, hexane : ethyl acetate and ethyl acetate: methanol. Aliquots of all fractions were dried and dissolved in DMSO for bioassay. The fractions from 39 to 48 demonstrated activity, so they were combined $(4 \mathrm{~g})$ and then the extract was loaded on silica gel 
column again with the same solvents for elution, each step ten tubes of $15 \mathrm{ml}$. Three groups of fractions showed activity: $50-57,59-62$, and $64-80$. Every group of fractions was subjected to reversed-phase high-performance liquid chromatography equipped with a UV detector (Shimadzu, Japan). The gradient solution system was started from $50 \%$ acetonitrile $(0,1 \%$ TFA) and $50 \%$ deionized water $(0,1 \%$ TFA) over $10 \mathrm{~min}$ and then up to $100 \%$ acetonitrile $(0,1 \%$ TFA) in $30 \mathrm{~min}$ and maintained $20 \mathrm{~min}$ at $100 \%$. Flow rate: $4 \mathrm{ml} \mathrm{min}{ }^{-1}$; injection volume: $2 \mathrm{ml}$; a SunFire prep. C18 column $(5 \mu \mathrm{m}, 10 \times 250 \mathrm{~mm})$; and detector wavelengths: 214 and $254 \mathrm{~nm}$. There were collected 60 fractions of $4 \mathrm{ml}$ per minute and then they were tested. All groups of fractions contained the same active peak at 32 minutes (grindelic acid (1)).

2.2. Compounds Identification. Proton nuclear magnetic resonance ( $\left.{ }^{1} \mathrm{H} \mathrm{NMR}\right)$, carbon-13 nuclear magnetic resonance $\left({ }^{13} \mathrm{C} \mathrm{NMR}\right),{ }^{1} \mathrm{H}-{ }^{1} \mathrm{H}$ correlation spectroscopy (COSY), heteronuclear multiple-bond correlation (HMBC), and heteronuclear single quantum correlation (HSQC) spectra were measured on a Bruker 500 SB UltraShield ${ }^{\mathrm{TM}}$ plus NMR spectrometer, operating at $400 \mathrm{MHz}$ for ${ }^{1} \mathrm{H}$ and $100 \mathrm{MHz}$ for ${ }^{13} \mathrm{C}$. The determination of the melting point of the isolated compounds was carried out with OptiMelt Stanford Device.

2.2.1. Compound 1 (Grindelic Acid). Colorless transparent crystals (70.8 mg). M.p. 102-104 ${ }^{\circ} \mathrm{C}$. HRMS (FTMS + pESI) $\mathrm{m} / z$, calcd. for $\mathrm{C}_{20} \mathrm{H}_{32} \mathrm{O}_{3}[\mathrm{M}-\mathrm{H}]^{-}:$319.2351, found 319.2329. Degree of unsaturation -5 .

${ }^{1} \mathrm{H}$ NMR $\left(400 \mathrm{MHz}, \mathrm{CDCl}_{3}\right): \delta 0.79\left(\mathrm{H} 3, \mathrm{~s},-\mathrm{CH}_{3}-20\right)$, $\delta 0.84\left(\mathrm{H} 3, \mathrm{~s},-\mathrm{CH}_{3}-18\right), \delta 0.87\left(\mathrm{H} 3, \mathrm{~s},-\mathrm{CH}_{3}-19\right), \delta 1.35 \overline{(\mathrm{H}} 4, \mathrm{~s}$,$\left.\mathrm{CH}_{3}-16, \delta 1.73 \overline{(\mathrm{H} 3}, \mathrm{s},-\mathrm{CH}_{3}-17\right), \delta 2.66[\mathrm{H} 1, \mathrm{~d}(J=15.4 \mathrm{~Hz})$, $-\mathrm{H}-14 \mathrm{a}$ ], $\delta 2.53$ [H1, d $(J=15.4 \mathrm{~Hz}),-\mathrm{H}-14 \mathrm{~b}], \delta 5.58(\mathrm{H} 1, \mathrm{~s}$, H-7).

${ }^{13} \mathrm{C}-\mathrm{NMR}\left(100 \mathrm{MHz}, \mathrm{CDCl}_{3}\right): 32.8$ (C-1), 18.7 (C-2), 41.9 (C-3), 33.3 (C-4), 42.9 (C-5), 24.3 (C-6), 129.0 (C-7), 133.0 (C-8), 92.9 (C-9), 40.9 (C-10), 27.6 (C-11), 39.6 (C-12), 81.2 (C-13),47.5 (C-14), 172.1 (C-15), 26.9 (C-16), 21.4 (C-17), 33.0 (C-18), 22.1 (C-19), 16.8 (C-20).

COSY: $-\mathrm{H}(14 \mathrm{a}) \longrightarrow-\mathrm{H}(14 \mathrm{~b})$.

HSQC: $-\mathrm{CH}_{3}(20) \longrightarrow-\mathrm{CH}_{3}(20),-\mathrm{CH}_{3}(18) \longrightarrow-\mathrm{CH}_{3}$ (18), $-\underline{\mathrm{CH}}_{3}(19) \longrightarrow-\underline{\mathrm{CH}}_{3}(\overline{19}),-\underline{\mathrm{CH}}_{3}(17) \longrightarrow-\underline{\mathrm{CH}}_{3}(17)$, $-\underline{\mathrm{CH}}_{3}(16) \longrightarrow-\mathrm{CH}_{3}(1 \overline{6}), \mathrm{H}(14 \mathrm{a},(\mathrm{b}) \longrightarrow \mathrm{C}(14), \mathrm{H}(5) \longrightarrow \mathrm{C}$ $(5), \mathrm{H}(7) \longrightarrow \overline{\mathrm{C}}(7)$.

HMBC: $-\mathrm{CH}_{3}(20) \longrightarrow \mathrm{C}(10), \mathrm{C}(1), \mathrm{C}(8),-\mathrm{CH}_{3}(18) \longrightarrow$ $\mathrm{C}(3), \mathrm{C}(19), \mathrm{C}(4)),-\mathrm{CH}_{3}(19) \longrightarrow \mathrm{C}(4), \mathrm{C}(3),-\overline{\mathrm{CH}}_{3}(17) \longrightarrow$ $\mathrm{C}(9), \mathrm{C}(8), \mathrm{C}(7)),-\mathrm{CH}_{3}(16) \longrightarrow \mathrm{C}(12), \mathrm{C}(13), \mathrm{C}(14),-\mathrm{H}$ $(14 \mathrm{a}) \longrightarrow \mathrm{C}(16) \mathrm{C}(13), \mathrm{C}(15),-\mathrm{H}(14 \mathrm{~b}) \longrightarrow \mathrm{C}(16) \mathrm{C}(15) \mathrm{C}$ (13), C (12).

2.2.2. Compound 2 (5-Hydroxy-3,3', $4^{\prime}, 7$-tetramethoxyflavone, Retusine). Yellow needles (31 mg). M.p. $156-157^{\circ} \mathrm{C}$. (lit. $160-162^{\circ} \mathrm{C}$ ). HRMS (FTMS $+\mathrm{pESI}$ ) $\mathrm{m} / z$, calcd. for $\mathrm{C}_{19} \mathrm{H}_{18} \mathrm{O}_{7}[\mathrm{M}+\mathrm{H}]^{+}:$358.1130, found: 359.1131. Degree of unsaturation -11 .
${ }^{1} \mathrm{H}$ NMR $\left(400 \mathrm{MHz}, \mathrm{CDCl}_{3}\right): \delta 6.37[\mathrm{H1}, \mathrm{d}(J=2.5 \mathrm{~Hz})$, H-6], $\delta 6.45$ [H1, d $(J=2.5 \mathrm{~Hz}) \mathrm{H}-8$ ] $\delta 6.98$ (H1, s, H-5'), $\delta$ $7.75\left(\mathrm{H} 2, \mathrm{~m}, \mathrm{H}-2^{\prime}, 6^{\prime}\right), \delta 12.7(\mathrm{H} 1, \mathrm{~s}, 5-\mathrm{OH}), \delta 3.87(\mathrm{H} 3, \mathrm{~s}$, $\left.-\mathrm{OCH}_{3}\right), \delta 3.88\left(\mathrm{H} 3, \mathrm{~s},-\mathrm{OCH}_{3}\right), \delta 3.90\left(\mathrm{H} 3, \mathrm{~s},-\mathrm{OCH}_{3}\right), \delta 3.91$ $\left(\mathrm{H} 3, \mathrm{~s},-\mathrm{OCH}_{3}\right)$.

${ }^{13} \mathrm{C}-\mathrm{NMR}$ (100 MHz, DMSO): 148.9 (C-2), 139.1 (C-3), 178.9 (C-4), 162.2 (C-5), 98.0 (C-6), 165.1 (C-7), 92.4 (C-8), 156.0 (C-9), 106.2 (C-10), 123.1 (C-1), 111.0 (C-2), 156.9

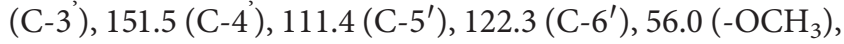
$56.2\left(-\mathrm{OCH}_{3}\right), 56.2\left(-\mathrm{OCH}_{3}\right), 60.4\left(-\mathrm{OCH}_{3}\right)$.

2.2.3. Compound $3\left(5,4^{\prime}\right.$-Dihydroxy-3,7,3'-trimethoxyflavone, Quercetin, 3,7,3'-Trimethyl Ether, Pachipodol). Yellow powder (20 mg). M.p. $160-162^{\circ} \mathrm{C}$. HRMS (FTMS + pESI) $\mathrm{m} / z$, calcd. for $\mathrm{C}_{18} \mathrm{H}_{16} \mathrm{O}_{7}[\mathrm{M}-\mathrm{H}]^{-}:$: 343.0823, found: 343.0825. Degree of unsaturation -11 .

${ }^{1} \mathrm{H}$ NMR (400 MHz, DMSO): $\delta 6.34[\mathrm{H} 1, \mathrm{~d}(J=2.2 \mathrm{~Hz})$, H-6], $\quad \delta \quad 6.43 \quad[\mathrm{H} 1, \quad \mathrm{~d} \quad(J=2.2 \mathrm{~Hz}), \quad \mathrm{H}-8], \quad \delta \quad 7.68$ [H1, d $(J=1.85 \mathrm{~Hz}), \mathrm{H}-2$ '] $\delta 7.65[\mathrm{H} 1, \mathrm{dd}(J=8.4,2.1 \mathrm{~Hz})$, H-6'], $\delta 7.03$ [H1, d $\left.(J=8.4 \mathrm{~Hz}), \mathrm{H}-5^{\prime}\right], \delta 3.84\left(\mathrm{H} 3, \mathrm{~s},-\mathrm{OCH}_{3}-\right.$ 3), $\delta 3.86\left(\mathrm{H} 3, \mathrm{~s},-\mathrm{OCH}_{3}-7\right), \delta 3.97\left(3 \mathrm{H}, \mathrm{s},-\mathrm{OCH}_{3}-3^{\prime}\right), \delta 12.64$ $(1 \mathrm{H}, \mathrm{s}, \mathrm{OH}-5), \delta 6.03\left(1 \mathrm{H}, \mathrm{s}, \mathrm{OH}-4^{\prime}\right)$.

${ }^{13} \mathrm{C}-\mathrm{NMR}$ (100 MHz, DMSO): 156.8 (C-2), 138.9 (C-3), 178.8 (C-4), 162.1 (C-5), 97.9 (C-6), 165.5 (C-7), 92.3 (C-8), 156.1 (C-9), 106.1 (C-10), 122.5 (C-1), 110.9 (C-2), 146.4 (C-3), $148.4(\mathrm{C}-4), 114.5\left(\mathrm{C}-5^{\prime}\right), 122.8\left(\mathrm{C}-6^{\prime}\right), 60.2\left(-\mathrm{OCH}_{3}-\right.$ 3), $55.9\left(-\mathrm{OCH}_{3}-7\right), 56.2\left(-\mathrm{OCH}_{3}-3^{\prime}\right)$.

COSY: $\mathrm{H}-5 \rightarrow \mathrm{H}-6$.

HSQC: $\mathrm{H}(6) \longrightarrow \mathrm{C}(6), \mathrm{H}(8) \longrightarrow \mathrm{C}(8), \mathrm{H}\left(2^{\prime}\right) \longrightarrow \mathrm{C}\left(2^{\prime}\right)$, $\mathrm{H}\left(5^{\prime}\right) \longrightarrow \mathrm{C}\left(5^{\prime}\right), \mathrm{H}\left(6^{\prime}\right) \longrightarrow \mathrm{C}\left(6^{\prime}\right),-\mathrm{OCH}_{3}(3) \longrightarrow \mathrm{OCH}_{3}(3)$, $-\mathrm{OCH}_{3}(7) \longrightarrow-\mathrm{OCH}_{3}(7),-\mathrm{OCH}_{3}\left(3^{\prime}\right) \longrightarrow-\mathrm{OCH}_{3}\left(3^{\prime}\right)$.

HMBC: $-\mathrm{OCH}_{3}(7) \longrightarrow \mathrm{C}(\overline{7}),-\mathrm{OCH}_{3}(3) \longrightarrow \mathrm{C}(3)$, $-\mathrm{OCH}_{3}\left(3^{\prime}\right) \longrightarrow \mathrm{C}\left(3^{\prime}\right),-\mathrm{H}(6) \longrightarrow \mathrm{C}(5), \overline{\mathrm{C}}(7), \mathrm{C}(10),-\mathrm{H}$ $(8) \longrightarrow \mathrm{C}(7), \mathrm{C}(9), \mathrm{C}(6), \mathrm{C}(10),-\mathrm{H}\left(2^{\prime}\right) \longrightarrow \mathrm{C}\left(6^{\prime}\right), \mathrm{C}\left(4^{\prime}\right)$, $\mathrm{C}(2),-\mathrm{H}\left(5^{\prime}\right) \longrightarrow \mathrm{C}\left(1^{\prime}\right), \mathrm{C}\left(3^{\prime}\right),-\mathrm{H}\left(6^{\prime}\right) \longrightarrow \mathrm{C}\left(2^{\prime}\right), \mathrm{C}\left(4^{\prime}\right)$, $-\mathrm{OH}(5) \longrightarrow \mathrm{C}(5), \mathrm{C}(6), \mathrm{C}(10)$.

2.2.4. Compound 4 (5, $4^{\prime}$-Dihydroxy-3,7-dimethoxyflavone, Jaranol). Yellow powder (70.8 mg). M.p. $230-233^{\circ} \mathrm{C}$. (lit. m.p. $\left.235-236^{\circ} \mathrm{C}\right) . \mathrm{C}_{17} \mathrm{H}_{14} \mathrm{O}_{6} .$. Degree of unsaturation $-11 .{ }^{1} \mathrm{H}$ NMR (400 MHz, DMSO): $\delta 6.32$ (H1, s, H-6), $\delta 6.70(\mathrm{H} 1, \mathrm{~s}$, $\mathrm{H}-8), \quad \delta 6.91 \quad\left[\mathrm{H} 2, \quad \mathrm{~d} \quad(J=9.16 \mathrm{~Hz}), \quad \mathrm{H}-3^{\prime}, \quad 5^{\prime}\right], \quad \delta 7.93$ $\left[\mathrm{H} 2, \mathrm{~d}(J=9.16 \mathrm{~Hz}), \mathrm{H}-2^{\prime}, 6^{\prime}\right], \delta 3.74\left(\mathrm{H} 3, \mathrm{~s},-\mathrm{OCH}_{3}-3\right), \delta 3.81$ $\left(\mathrm{H} 3, \mathrm{~s},-\mathrm{OCH}_{3}-7\right), \delta 10.37\left(\mathrm{H} 1, \mathrm{~s},-\mathrm{O} \underline{\mathrm{H}}-4^{\prime}\right), \delta 12.62(\mathrm{H} 1, \mathrm{~s}$, $-\mathrm{OH}-5)$.

${ }^{13} \mathrm{C}-\mathrm{NMR}$ (100 MHz, DMSO): 156.5 (C-2), 138.3 (C-3), 178.6 (C-4), 161.4 (C-5), 98.3 (C-6), 165.6 (C-7), 92.9 (C-8), 156.8 (C-9), 105.7 (C-10), 121.0 (C-1), 130.8 (C-2, 6' ), 116.2 $\left(\mathrm{C}-3,5^{\prime}\right), 160.8\left(\mathrm{C}-4^{\prime}\right), 60.3\left(-\mathrm{OCH}_{3}-3\right), 56.6\left(-\mathrm{OCH}_{3}-7\right)$. COSY: $-\mathrm{H}(6) \longrightarrow-\mathrm{H}(8),-\mathrm{H}(8) \longrightarrow-\mathrm{H}(6),-\mathrm{H}\left(2^{\prime}, 6^{\prime}\right) \longrightarrow$ $-\mathrm{H}\left(3^{\prime}, 5^{\prime}\right),-\mathrm{H}\left(3^{\prime}, 5^{\prime}\right) \longrightarrow-\mathrm{H}\left(2^{\prime}, 6^{\prime}\right)$.

HSQC: $\mathrm{H}(6) \longrightarrow \mathrm{C}(6), \mathrm{H}(8) \longrightarrow \mathrm{C}(8), \mathrm{H}\left(2^{\prime}, 6^{\prime}\right) \longrightarrow \mathrm{C}$ $\left(2^{\prime}, 6^{\prime}\right), \mathrm{H}\left(3^{\prime}, 5^{\prime}\right) \longrightarrow \mathrm{C}\left(3^{\prime}, 5^{\prime}\right),-\mathrm{OCH}_{3}(3) \longrightarrow-\mathrm{OCH}_{3}(3)$, $-\mathrm{OCH}_{3}(7) \longrightarrow-\mathrm{OCH}_{3}(7)$.

HMBC: $-\mathrm{OCH}_{3}(3) \longrightarrow \mathrm{C}(3),-\mathrm{OCH}_{3}(7) \longrightarrow \mathrm{C}(7),-\mathrm{H}$ (6) $\longrightarrow \mathrm{C}(5) \mathrm{C}(\overline{10}),-\mathrm{H}(8) \longrightarrow \mathrm{C}(6) \mathrm{C}(10) \mathrm{C}(9) \mathrm{C}(7),-\mathrm{H}$ $\left(2^{\prime}\right) \longrightarrow \mathrm{C}\left(6^{\prime}\right) \mathrm{C}(2) \mathrm{C}\left(4^{\prime}\right),-\mathrm{H}\left(6^{\prime}\right) \longrightarrow \mathrm{C}\left(2^{\prime}\right) \mathrm{C}(2) \mathrm{C}\left(4^{\prime}\right),-\mathrm{H}$ 
$\left(3^{\prime}\right) \longrightarrow \mathrm{C}\left(5^{\prime}\right) \mathrm{C}\left(1^{\prime}\right), \mathrm{C}\left(4^{\prime}\right), \mathrm{H}\left(5^{\prime}\right) \longrightarrow \mathrm{C}\left(3^{\prime}\right) \mathrm{C}\left(1^{\prime}\right), \mathrm{C}\left(4^{\prime}\right)$ $-\mathrm{OH}\left(4^{\prime}\right) \longrightarrow \mathrm{C}\left(3^{\prime}\right) \mathrm{C}\left(5^{\prime}\right) \mathrm{C}\left(4^{\prime}\right)$.

2.2.5. Compound 5 (5,4'-Dihydroxy-3,6,7-trimethoxyflavone, Penduletin). Yellow powder $(216.8 \mathrm{mg})$. M.p. $210-212^{\circ} \mathrm{C}$. (lit. m.p. $216-218^{\circ} \mathrm{C}$ ). HRMS (FTMS + pESI) $\mathrm{m} / z$, calcd. for $\mathrm{C}_{18} \mathrm{H}_{16} \mathrm{O}_{7}[\mathrm{M}+\mathrm{H}]^{+}:$345.0974, found:345.0969. Degree of unsaturation -11 .

${ }^{1} \mathrm{H}$ NMR (400 MHz, DMSO): $\delta 6.92$ (H1, s, H-8), $\delta 6.955$ [H2, d $(J=8.8 \mathrm{~Hz}) \mathrm{H}-3,5], \delta 7.99[\mathrm{H} 2, \mathrm{~d}(J=8.8 \mathrm{~Hz}) \mathrm{H}-2$, 6], $\delta 3.73\left(\mathrm{H} 3, \mathrm{~s},-\mathrm{OCH}_{3}\right), \delta 3.80\left(\mathrm{H} 3, \mathrm{~s},-\mathrm{OCH}_{3}\right), \delta 3.92(\mathrm{H} 3$, s, $\left.-\mathrm{OCH}_{3}\right)$.

${ }^{13}$ C-NMR (100 MHz, DMSO): 152.3 (C-2), 159.1 (C-3), 178.1 (C-4), 156.40 (C-5), 138.1 (C-6), 132.1 (C-7), 91.9 (C8), 152.2 (C-9), 106.0 (C-10), 121.0 (C-1), 130.7 (C-2, 6), $116.1(\mathrm{C}-3,5), 160.8(\mathrm{C}-4), 57.0\left(-\mathrm{OCH}_{3}-3\right), 60.2\left(-\mathrm{OCH}_{3}-\right.$ 6), $60.5\left(-\mathrm{OCH}_{3}-7\right)$. $\left.5^{\prime}\right)$

COSY: $-\mathrm{H}\left(3^{\prime}, 5^{\prime}\right) \longrightarrow-\mathrm{H}\left(2^{\prime}, 6^{\prime}\right),-\mathrm{H}\left(2^{\prime}, 6^{\prime}\right) \longrightarrow-\mathrm{H}\left(3^{\prime}\right.$,

HSQC: $\mathrm{H}(8) \longrightarrow \mathrm{C}(8), \mathrm{H}\left(3^{\prime}, 5^{\prime}\right) \longrightarrow \mathrm{C}\left(3^{\prime}, 5^{\prime}\right), \mathrm{H}\left(2^{\prime}\right.$, $\left.6^{\prime}\right) \longrightarrow \mathrm{C}\left(2^{\prime}, 6^{\prime}\right),-\mathrm{OCH}_{3}(7) \longrightarrow \mathrm{OCH}_{3}(7),-\mathrm{OCH}_{3}(6) \longrightarrow$ $-\mathrm{OCH}_{3}(6),-\mathrm{OCH}_{3}(3) \longrightarrow-\mathrm{OCH}_{3}$ (3).

$\mathrm{HMBC}:-\mathrm{OCH}_{3}(7) \longrightarrow \mathrm{C}(7),-\mathrm{OCH}_{3}(6) \longrightarrow \mathrm{C}(6)$, $-\mathrm{OCH}_{3}(3) \longrightarrow \mathrm{C}(3),-\mathrm{H}(8) \longrightarrow \mathrm{C}(7) \mathrm{C}(9) \mathrm{C}(10),-\mathrm{H}\left(3^{\prime}\right.$, $\left.5^{\prime}\right) \longrightarrow \mathrm{C}\left(1^{\prime}\right),-\mathrm{H}\left(2^{\prime}, 6^{\prime}\right) \longrightarrow \mathrm{C}\left(4^{\prime}\right),-\mathrm{OH}\left(4^{\prime}\right) \longrightarrow \mathrm{C}\left(4^{\prime}\right)$.

2.2.6. Compound 6 (5,3'-Dihyroxy-3,6,7,4'-tetramethoxyflavone, Quercetagetin, 3,6,7-Trimethyl Ether, Casticin). Yellow powder (403.3 mg). M.p. $186.4-187^{\circ} \mathrm{C}$. HRMS (FTMS + pESI) $\mathrm{m} / z$, calcd. for $\mathrm{C}_{19} \mathrm{H}_{18} \mathrm{O}_{8}[\mathrm{M}+\mathrm{H}]^{+}: 375.1079$, found: 375.1074. Degree of unsaturation -11 .

${ }^{1} \mathrm{H}$ NMR (400 MHz, DMSO): $\delta 6.87(\mathrm{H1}, \mathrm{s}, \mathrm{H}-8), \delta 7.11$ [H1, d $\left.(J=9.16 \mathrm{~Hz}) \mathrm{H}-5^{\prime}\right], \delta 7.59\left(\mathrm{H} 2, \mathrm{~m}, \mathrm{H}-2^{\prime}, 6\right), \delta 3.75$ $\left(\mathrm{H} 3, \mathrm{~s},-\mathrm{OCH}_{3}-6\right), \delta 3.81\left(\mathrm{H} 3, \mathrm{~s},-\mathrm{OCH}_{3}-3\right), \delta 3.88(\mathrm{H} 3, \mathrm{~s}$, $\left.-\mathrm{OCH}_{3}-4^{\prime}\right), \delta 3.93\left(\mathrm{H} 3, \mathrm{~s},-\mathrm{OCH}_{3}-7\right), \delta 9.32\left(\mathrm{H} 1, \mathrm{~s},-\mathrm{OH}-3^{\prime}\right)$, $\delta 12.59(\mathrm{H} 1, \mathrm{~s},-\mathrm{OH}-5)$.

${ }^{13} \mathrm{C}-\mathrm{NMR}(100 \overline{\mathrm{MHz}}, \mathrm{DMSO}): 152.2$ (C-2), 138.6 (C-3), 178.8 (C-4), 152.3 (C-5), 132.3 (C-6), 159.2 (C-7), 91.9 (C-8), 156.2 (C-9), 106.2 (C-10), 122.8 (C-1), 115.7 (C-2), 147.0 $(\mathrm{C}-3), \quad 150.9$ (C-4), $112.5 \quad\left(\mathrm{C}-5^{\prime}\right), 120.9 \quad\left(\mathrm{C}-6^{\prime}\right), 60.5$ $\left(-\mathrm{OCH}_{3}-6\right), 57.0\left(-\mathrm{OCH}_{3}-4^{\prime}\right), 60.2\left(-\mathrm{OCH}_{3}-3\right), 56.1\left(-\mathrm{OCH}_{3}-\right.$ 7).

COSY: $\mathrm{H}-5^{\prime} \longrightarrow \mathrm{H}-6$.

HSQC: $\mathrm{H}(8) \longrightarrow \mathrm{C}(8), \mathrm{H}\left(2^{\prime}\right) \longrightarrow \mathrm{C}\left(2^{\prime}\right), \mathrm{H}\left(5^{\prime}\right) \longrightarrow \mathrm{C}$ $\left(5^{\prime}\right), \mathrm{H}\left(6^{\prime}\right) \longrightarrow \mathrm{C}\left(6^{\prime}\right),-\mathrm{OCH}_{3}(7) \longrightarrow-\mathrm{OCH}_{3}(7),-\mathrm{OCH}_{3}$ $(6) \longrightarrow-\mathrm{OCH}_{3}$ (6), $-\mathrm{OCH}_{3}$ (3) $\longrightarrow-\underline{\mathrm{OCH}}_{3}$ (3), $-\underline{\mathrm{OC}}_{3}$ $\left(4^{\prime}\right) \longrightarrow-\mathrm{OCH}_{3}\left(4^{\prime}\right)$.

$\mathrm{HMBC}:-\mathrm{OCH}_{3}(6) \longrightarrow \mathrm{C}(6),-\mathrm{OCH}_{3}(7) \longrightarrow \mathrm{C}(7)$, $-\mathrm{OCH}_{3}(3) \longrightarrow \mathrm{C}(3),-\mathrm{OCH}_{3}\left(4^{\prime}\right) \longrightarrow \mathrm{C}\left(4^{\prime}\right),-\mathrm{H}(8) \longrightarrow \mathrm{C}(6)$ $\mathrm{C}(7) \mathrm{C}(2)(10),-\mathrm{H}\left(5^{\prime}\right) \longrightarrow \mathrm{C}\left(1^{\prime}\right) \mathrm{C}\left(3^{\prime}\right),-\mathrm{OH}(5) \rightarrow \mathrm{C}$ (5) C (6) C (10).

2.2.7. Compound $7 \quad$ (5,7,3'-Trihydroxy-3, $4^{\prime}$ dimethoxyflavone). Yellow powder (294.8 mg). M.p. 228-230 ${ }^{\circ} \mathrm{C}$. (lit. m.p. 235-236 ${ }^{\circ} \mathrm{C}$ ). HRMS (FTMS + pESI) m/ $z$, calcd. for $\mathrm{C}_{17} \mathrm{H}_{14} \mathrm{O}_{7}[\mathrm{M}+\mathrm{H}]^{+}$: 331.0817, found: 331.0812 . Degree of unsaturation -11 .
${ }^{1} \mathrm{H}$ NMR (400 MHz, DMSO): $\delta 6.21[\mathrm{H} 1, \mathrm{~d}(J=1.7 \mathrm{~Hz})$, H-6], $\delta \quad 6.43 \quad[\mathrm{H} 1, \quad \mathrm{~d} \quad(J=2.3 \mathrm{~Hz}), \quad \mathrm{H}-8], \quad \delta \quad 7.11$ $\left[\mathrm{H} 1, \mathrm{~d}(J=8.6 \mathrm{~Hz}) \mathrm{H}-5^{\prime}\right], \delta 7.54(\mathrm{H} 1, \mathrm{~s}, \mathrm{H}-2), \delta 7.56$ $\left[\mathrm{H} 1, \mathrm{~d}(J=8.6 \mathrm{~Hz}), \mathrm{H}-6^{\prime}\right], \delta 3.86\left(\mathrm{H} 3, \mathrm{~s},-\mathrm{OCH}_{3}-4^{\prime}\right), \delta 3.79$ $\left(\mathrm{H} 3, \mathrm{~s},-\mathrm{OCH}_{3}-3\right), \delta 12.67(\mathrm{H} 1, \mathrm{~s},-\mathrm{OH}-5), \delta 9.6(\mathrm{H} 1, \mathrm{~s},-\mathrm{OH})$, $\delta 10.9(\mathrm{H} 1, \mathrm{~s},-\mathrm{OH})$.

${ }^{13} \mathrm{C}-\mathrm{NMR}$ (100 MHz, DMSO): 155.3 (C-2), 138.0 (C-3), 178.0 (C-4), 161.3 (C-5), 98.6 (C-6), 164.2 (C-7), 93.7 (C-8), 156.4 (C-9), 104.3 (C-10), 122.3 (C-1), 115.0 (C-2), 146.4 (C-3), $150.2(\mathrm{C}-4), 111.9\left({\mathrm{C}-5^{\prime}}^{\prime}\right), 120.4\left(\mathrm{C}^{\prime} 6^{\prime}\right), 59.8\left(-\mathrm{OCH}_{3}-\right.$ 3), 55.6(- $\left.\mathrm{OCH}_{3}-4^{\prime}\right)$.

COSY: $-\mathrm{H}(6) \longrightarrow-\mathrm{H}(8),-\mathrm{H}(8) \longrightarrow-\mathrm{H}(6)$.

HSQC: $\mathrm{H}(6) \longrightarrow \mathrm{C}(6), \mathrm{H}(8) \longrightarrow \mathrm{C}(8), \mathrm{H}\left(5^{\prime}\right) \longrightarrow \mathrm{C}\left(5^{\prime}\right)$, $\mathrm{H}\left(2^{\prime}\right) \longrightarrow \mathrm{C}\left(2^{\prime}\right), \mathrm{H}\left(6^{\prime}\right) \longrightarrow \mathrm{C}\left(6^{\prime}\right),-\mathrm{OCH}_{3}(3) \longrightarrow-\mathrm{OCH}_{3}$ (3), $-\mathrm{OCH}_{3}\left(4^{\prime}\right) \longrightarrow-\mathrm{OCH}_{3}\left(4^{\prime}\right)$.

$\mathrm{HMBC}:-\mathrm{OCH}_{3}(3) \longrightarrow \mathrm{C}(3),-\mathrm{OCH}_{3}\left(4^{\prime}\right) \longrightarrow \mathrm{C}\left(4^{\prime}\right),-\mathrm{H}$ $(6) \longrightarrow \mathrm{C}(8) \mathrm{C}(10),-\mathrm{H}(8) \longrightarrow \mathrm{C}(6) \mathrm{C}(10),-\mathrm{H}\left(5^{\prime}\right) \longrightarrow \mathrm{C}\left(1^{\prime}\right)$ $\mathrm{C}\left(3^{\prime}\right),-\mathrm{H}\left(2^{\prime}\right) \longrightarrow \mathrm{C}\left(6^{\prime}\right) \mathrm{C}\left(4^{\prime}\right) \mathrm{C}(2) \mathrm{C}\left(3^{\prime}\right),-\mathrm{H}\left(6^{\prime}\right) \longrightarrow \mathrm{C}$ $\left(2^{\prime}\right),-\mathrm{OH}(5) \longrightarrow \mathrm{C}(5) \mathrm{C}(6) \mathrm{C}(10)$.

2.3. Determination of the Cytotoxic Activity. The $55 \mathrm{ml}$ separatory funnel was filled with artificial seawater and added $200 \mathrm{mg}$ eggs of Artemia salina. Then, it was kept with a soft supply of air for three days until the crustaceans hatch from nauplii. One side of the funnel was covered with aluminum foil, and after 5 minutes, the larvae, which moved on the bright side of the separatory funnel, were removed with a Pasteur pipette.

20-40 nauplii were placed into each of the 24 microtiter plates with $990 \mu \mathrm{l}$ of seawater. Dead larvae were counted under a microscope. $10 \mu \mathrm{l}$ of dimethylsulfoxide solution per $10 \mathrm{mg} \mathrm{ml}^{-1}$ sample was added. Actinomycin D or staurosporine was used as a standard comparison reagent, and DMSO was a negative control. After $24 \mathrm{~h}$ of incubation and further maintaining microtiter plates for 24 hours (to ensure immobility) the dead nauplii were counted under the microscope.

Mortality $\mathrm{P}$ was determined by the following formula:

$$
\mathbf{P}=\frac{(\mathbf{A}-\mathbf{N}-\mathbf{B})}{\mathbf{Z}} * 100 \%,
$$

where $\mathbf{A}$ is the number of dead nauplii after $24 \mathrm{~h}$; $\mathbf{N}$ is the number of nauplii that died before the test; and $\mathbf{B}$ is the average amount of nauplii died in a negative control.

2.4. Antimicrobial Activity. The antimicrobial assay performed a broth microdilution method against 4 bacterial strains, i.e., Gram-positive Staphylococcus aureus 6532, Bacillus cereus, Gram-negative Salmonella enteritidis, and yeasted strain Candida albicans sc5314. In this assay, standard antibiotics (ampicillin $5 \mathrm{mg} \mathrm{ml}^{-1}$ for bacteria and fluconazole for Candida albicans) and DMSO/water were served as positive and negative controls for the sensitivity of the tested bacteria, respectively.

A single colony of bacteria was inoculated from an agar plate in $3 \mathrm{ml} \mathrm{MH}$ medium $(0.2 \%$ beef extract, $1.75 \%$ 
<smiles>CC1=CC[C@H]2[C@@H](C)CCC[C@]2(C)[C@]12CC[C@](C)(CC(=O)O)O2</smiles><smiles>COc1cc(O)c2c(=O)c(OC)c(-c3ccc(OC)c(OC)c3)oc2c1</smiles><smiles>COc1cc2oc(-c3ccc(O)cc3)c(OC)c(=O)c2c(O)c1OC</smiles>

2<smiles>COc1cc(O)c2c(=O)c(OC)c(-c3ccc(O)c(OC)c3)oc2c1</smiles>

3<smiles>COc1cc(O)c2c(=O)c(OC)c(-c3ccc(O)cc3)oc2c1</smiles>

4<smiles>COc1ccc(-c2oc3cc(OC)c(OC)c(O)c3c(=O)c2OC)cc1O</smiles>

6<smiles>COc1ccc(-c2oc3cc(O)cc(O)c3c(=O)c2OC)cc1O</smiles>

Figure 1: Chemical structures of the isolated compounds.

casamino acids, and $0.015 \%$ soluble starch) and in YPD medium ( $1 \%$ yeast extract, $2 \%$ peptone, and $2 \%$ dextrose) for C. albicans. The tubes were incubated overnight in a shaking incubator $(200 \mathrm{rpm})$ at $37^{\circ} \mathrm{C}$.

Ten $\mu \mathrm{L}$ of the test sample was transferred into the wells of a 96-well plate, as well as the positive control (ampicillin, stock $5 \mathrm{mg} / \mathrm{mL}$ ) and blank (solvent) controls (DMSO and water). Each well of a microdilution plate was then inoculated with $190 \mu \mathrm{L}$ of the diluted standardized inoculum $(\mathrm{OD}=0.003$ at $620 \mathrm{~nm})$. Control wells were prepared with $190 \mu \mathrm{L} \mathrm{MH}$ broth and $10 \mu \mathrm{L}$ extract to correct any absorption due to extracting components. The microdilution plates were placed in a shaker-incubator at $37^{\circ} \mathrm{C}$ for $24 \mathrm{~h}$ and then read on microplate reader at $620 \mathrm{~nm}$. The OD was measured at a wavelength of $620 \mathrm{~nm}$. The relative inhibition (\%) of the test sample was calculated by the following formula:

$$
\% \text { inh }=100-\left(\frac{\mathrm{A}-\mathrm{B}}{\mathrm{C}} * 100\right),
$$

where $A$ is the OD value of the sample; $B$ is the OD value of the noninoculated sample control; and $\mathrm{C}$ is the average $\mathrm{OD}$ value of the solvent (DMSO) [44].

2.5. Molecular Docking. The crystal structure of the target HDAC (PDB ID: 2VQM; resolution: 1.80 ̊) was downloaded from Protein Data Bank (http://www.pdb.org). Molecular Operating Environment (MOE) was used for the docking analysis [45]. In these studies, the free energies and binding mode of the examined molecule against HDAC were determined. At first, the water molecules were removed from the crystal structure of HDAC, retaining only one 
TABle 1: Antimicrobial activity of compounds (\% inhibition) compared to DMSO.

\begin{tabular}{|c|c|c|c|c|}
\hline Compound & S. aureus & B. cereus & S. enteritidis & C. albicans \\
\hline \multicolumn{5}{|l|}{$100 \mu \mathrm{g} \mathrm{ml}^{-1} / 50 \mu \mathrm{g} \mathrm{ml}^{-1}$} \\
\hline 1 & 92.3 & 73.6 & 39.5 & 51.9 \\
\hline 2 & 28.8 & 48.5 & -16.7 & 18.5 \\
\hline 3 & 28.1 & 45.7 & -3.6 & 1.3 \\
\hline 4 & 7.1 & 46.8 & 23.4 & 0.3 \\
\hline 5 & 8.5 & 22.7 & 8.3 & 13.2 \\
\hline 6 & 4 & 42.7 & 29.6 & 9.8 \\
\hline 7 & 16.6 & 22.5 & 26.7 & 9.5 \\
\hline Ampicillin $\left(250 \mu \mathrm{g} \mathrm{ml}^{-1}\right)$ & 95 & 90 & 78 & - \\
\hline Nystatin $\left(62.5 \mu \mathrm{g} \mathrm{ml}^{-1}\right)$ & - & - & - & 98 \\
\hline \multicolumn{5}{|l|}{$50 \mu \mathrm{g} \mathrm{ml}^{-1} / 25 \mu \mathrm{g} \mathrm{ml}^{-1}$} \\
\hline 1 & 76.7 & 0.71 & 12.7 & 42.5 \\
\hline 2 & -13 & 38.7 & -20 & 5.3 \\
\hline 3 & 10.7 & 19.7 & 2.6 & -7.7 \\
\hline 4 & -9.5 & 34 & -9.2 & -4.4 \\
\hline 5 & -15.77 & -19 & -45.5 & 4.4 \\
\hline 6 & -9.87 & 20.8 & -24.3 & 4 \\
\hline 7 & -7.9 & -46 & -38.8 & 3.2 \\
\hline \multicolumn{5}{|l|}{$25 \mu \mathrm{g} \mathrm{ml}^{-1} / 12.5 \mu \mathrm{g} \mathrm{ml}^{-1}$} \\
\hline 1 & 10.8 & 2.03 & -29.6 & 14.8 \\
\hline 2 & -27.4 & -43 & -30 & -3.2 \\
\hline 3 & 12.1 & 11.3 & 1.7 & -7.2 \\
\hline 4 & 18.4 & 3.2 & -8.6 & -3.2 \\
\hline 5 & -41 & -72 & -64.8 & 0.6 \\
\hline 6 & -34 & 2.2 & -39.3 & 0.3 \\
\hline 7 & -24 & -48 & -52.5 & 10.24 \\
\hline
\end{tabular}

chain and zinc atoms which are essential for binding. The cocrystallized ligand (hydroxamic acid derivative, HA3) was used as a reference ligand. Then, the protein structure was protonated and the hydrogen atoms were hidden. Next, the energy was minimized and the binding pocket of the protein was defined [46-48].

The structures of the examined compound and the cocrystallized ligand were drawn using ChemBioDraw Ultra 14.0 and saved in SDF format. Then, the saved file was opened using MOE software and 3D structures were protonated. Next, the energy of the molecules was minimized. The validation process was performed for the target receptor by running the docking process for only the cocrystallized ligand. Low RMSD values between docked and crystal conformations indicate valid performance [49, 50]. The docking procedures were carried out utilizing a default protocol. In each case, 10 docked structures were generated using genetic algorithm searches [51-53].

\section{Results and Discussion}

3.1. Isolation and Structure Elucidation. Compound 1 was isolated using biologically guided chromatography for the methanolic extract of C. alata aerial parts. Additionally, the chloroform extract was subjected to different chromatographic techniques to give six flavonoids (2-7). Structural identification of compounds was carried out by $1 \mathrm{D}$ and $2 \mathrm{D}$ NMR spectroscopy and then comparison with literature data. All compounds were isolated for the first time from plants of the Cousinia genus. The isolated compounds (Figure 1) were identified to be grindelic acid (1) [54], retusine (2) [55], pachipodol (3) [56], jaranol (4) [57], penduletin (5) [58], casticin (6) [59], and 5,7,3'-trihydroxy$3,4^{\prime}$-dimethoxyflavone (7) [60].

3.2. Cytotoxic Activity. The cytotoxic activity of the isolated compounds was determined using the brine shrimp (Artemia salina) lethality test. The results showed that, among the isolated compounds, grindelic acid (1) and penduletin (5) were able to inhibit the crustaceans in all tested concentrations $\left(10.0,5.0\right.$, and $\left.1.0 \mathrm{mg} \mathrm{ml}^{-1}\right)$ while grindelic acid (1) had the highest activity comparing with other isolated compounds. The average activity was showed by retusine (2) and 5, 7, 3' -trihydroxy-3, $4^{\prime}$-dimethoxyflavone (7); these compounds were active at $10-5 \mathrm{mg} \mathrm{ml}^{-1}$ and inactive at $1 \mathrm{mg} \mathrm{ml}^{-1}$. For retusine (2), the mortality was $96 \%$ and $83 \%$ at respective concentrations.

3.3. Antimicrobial Activity. Table 1 shows the results of the antimicrobial assay. It is worth noting that the concentration of samples for S. aureus, B. cereus, S. enteritidis ranged from 100 to $25 \mu \mathrm{g} \mathrm{ml}^{-1}$ and for C. albicans from 50 to $12.5 \mu \mathrm{g} \mathrm{ml}^{-1}$. Percentages of inhibition surpassing 50 were considered as an activity. None of the tested flavonoids (2-7) exhibited any activity, but the diterpene, grindelic acid (1), was active against Gram-positive bacterial strains S. aureus (at 100 and 


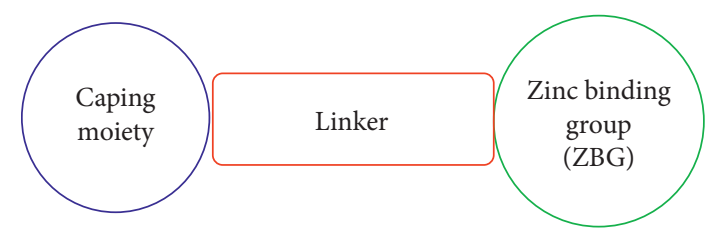

(a)

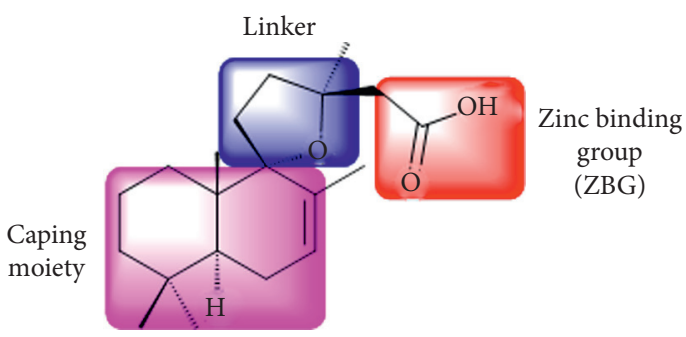

(b)

FIgURE 2: (a) Essential pharmacophoric features of HDAC inhibitors. (b) Essential pharmacophoric features of grindelic acid against HDAC.

TABLE 2: The docking binding free energies of compound 1 and the cocrystallized ligand (HA3) HDAC.

\section{Comp.} Binding free energy $(\mathrm{kcal} / \mathrm{mol})$

Cocrystallized ligand (HA3)

$-14.02$

1

$-18.70$

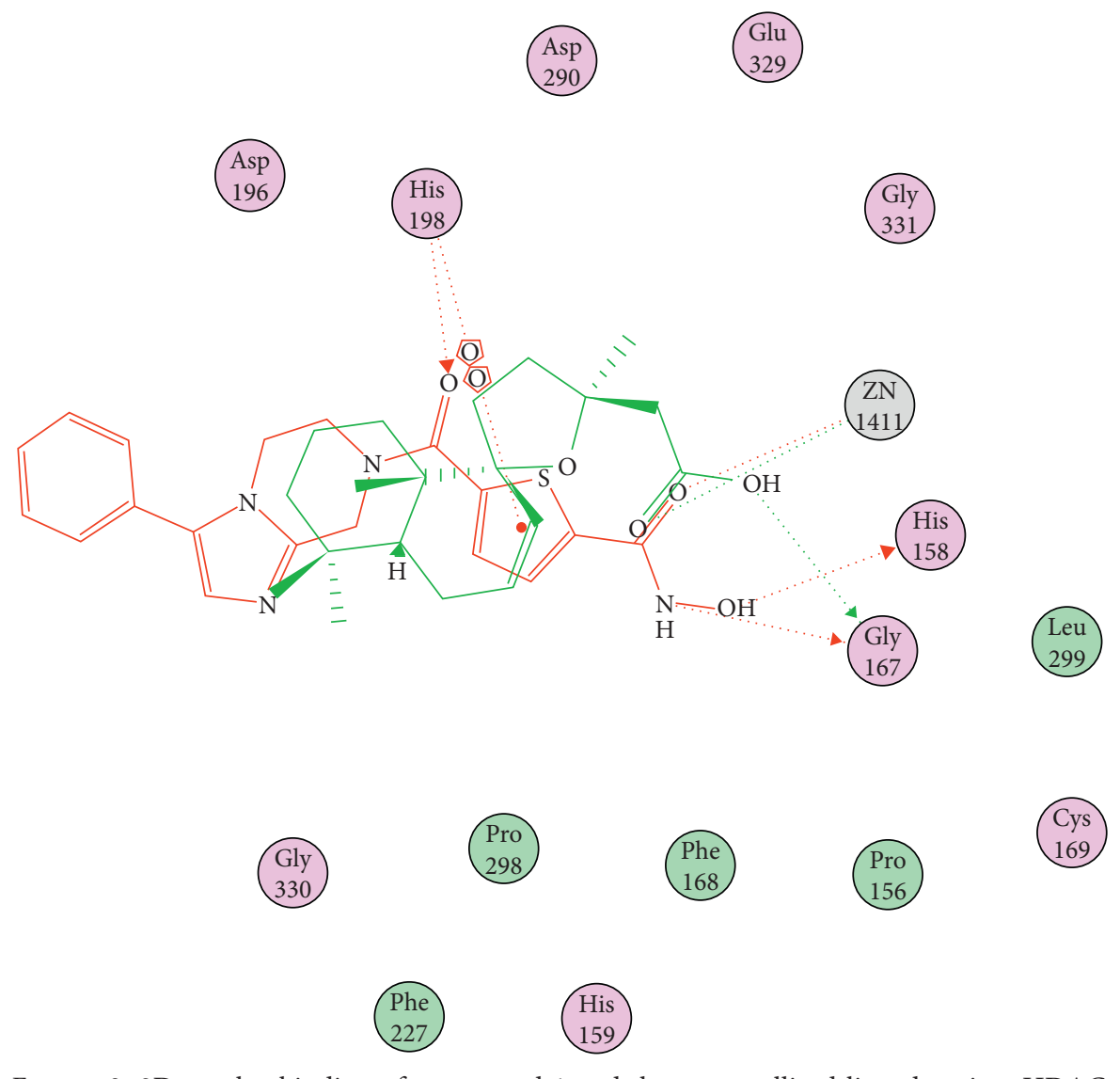

$50 \mu \mathrm{g} \mathrm{ml}^{-1}$ ), B. cereus (at $100 \mu \mathrm{g} \mathrm{ml}^{-1}$ ), and C. albicans (at $\left.50 \mu \mathrm{g} \mathrm{ml}^{-1}\right)$.

3.4. Molecular Docking. Three different reasons encouraged us to run a molecular docking study for the grindelic acid (1) against histone deacetylase (HDAC) enzyme. First is the promising cytotoxic and antibacterial results of grindelic acid which derived us to think deeper to find out the mechanism of action on a molecular level. Second is the presence of the essential pharmacophoric features in 


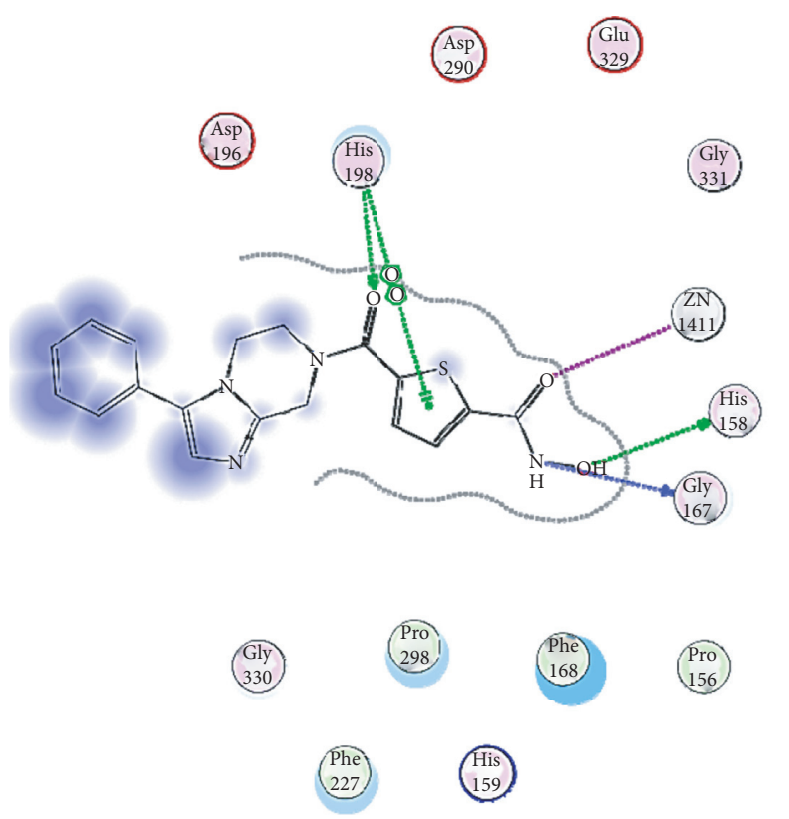

(a)

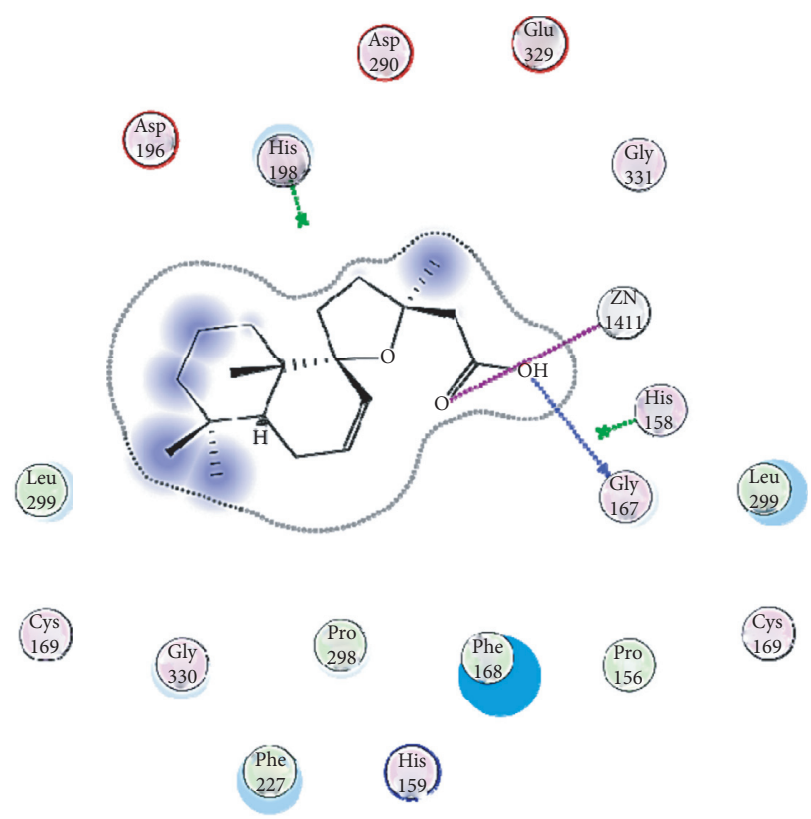

(b)

FIgURE 4: (a) 2D structure of the co-crystallized ligand docked into the active site of HDAC. (b) 2D structure of compound 1 docked into the active site of HDAC.

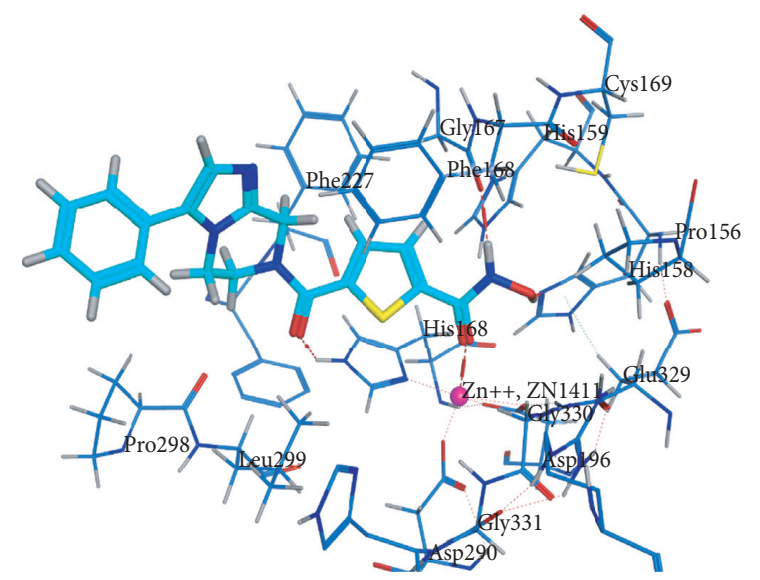

(a)

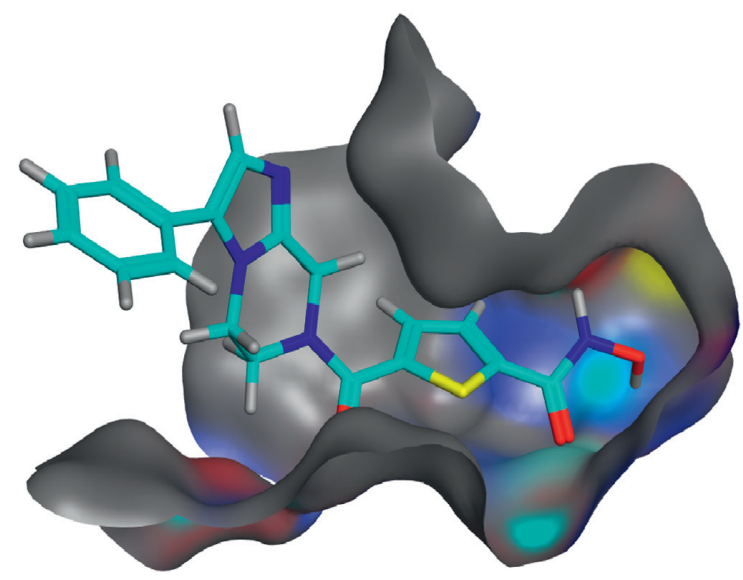

(b)

FIgURE 5: (a) 3D structure of the co-crystallized ligand docked into the active site of HDAC.(b) 3D structure of compound 1 docked into the active site of HDAC.

grindelic acid's chemical structure. Finally, the reported cytotoxic and antibacterial effects of HDAC inhibitors $[43,61]$. The reported histone deacetylase (HDAC) inhibitors must possess three main pharmacophoric features, comprising the zinc-binding group ( $\mathrm{ZBG}$ ) that chelated the zinc atom in the active site, a linker that accommodates the tubular access of the active site, and a capping group for interactions with the surface recognition motif connected by a small connecting unit to the linker (Figure 2(a)) $[62,63]$. All these features were found in grindelic acid (Figure 2(b)).

Docking studies were carried out for compound 1 against HDAC (PDB ID: 2VQM; resolution: $1.80 \AA$ ) to examine its mode of binding with the proposed target. The cocrystallized ligand (HA3) was used as a reference molecule. The results of docking studies revealed that the docked compound has a good binding affinity against HDAC with a binding free energy of $-18.70 \mathrm{kcal} / \mathrm{mol}$. Such compound exhibited a binding mode of interaction similar to that of the cocrystallized ligand (Table 2; Figure 3).

The crystallized ligand (HA3) showed a binding energy of $-14.02 \mathrm{kcal} / \mathrm{mol}$. The detailed binding mode of the crystallized ligand was as follows: the $N$-hydroxycarboxamide moiety occupied the zinc-binding region forming two hydrogen bonds with His158 and Gly167. Besides, it formed 


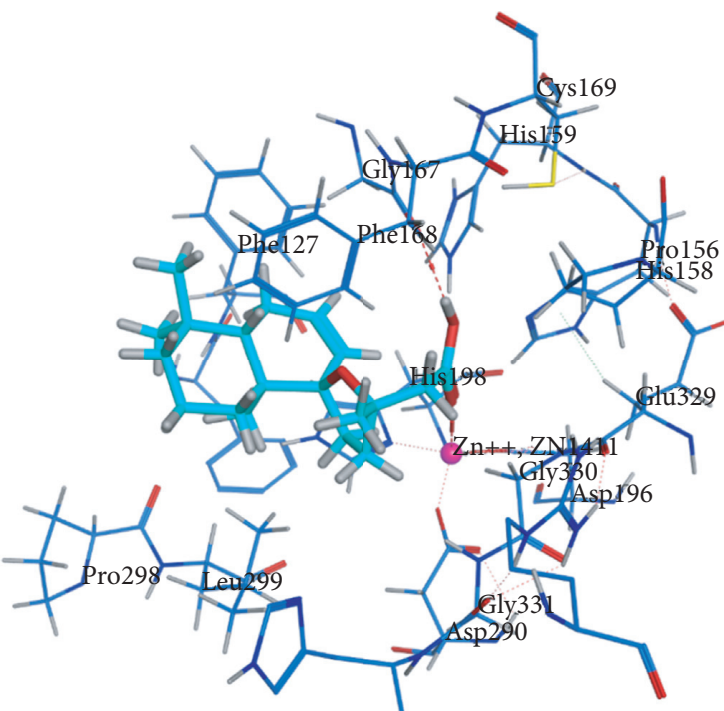

(a)

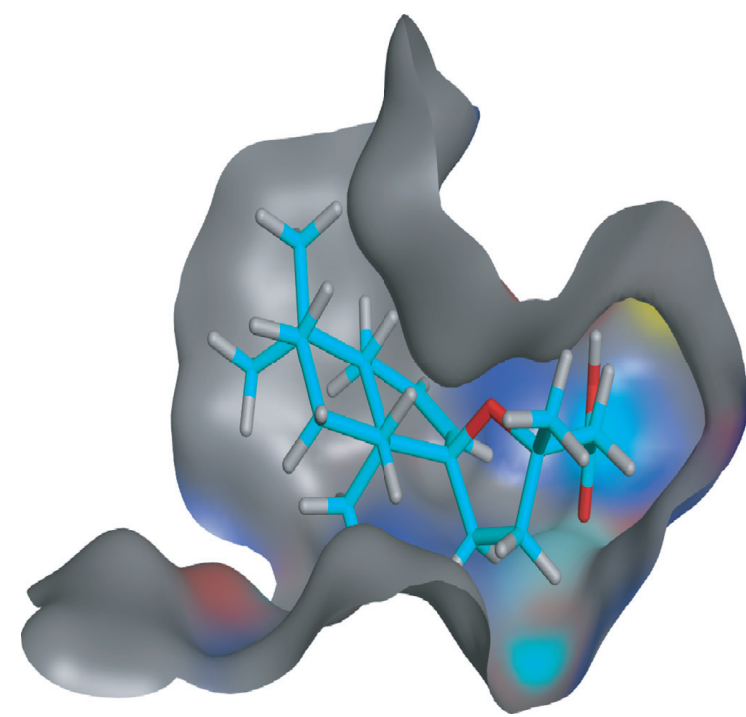

(b)

FIgURE 6: (a) 3D structure of compound 1 docked into the active site of HDAC (b) Mapping surface showing compound 1 occupying the active site of HDAC.

an electrostatic interaction with the Zn atom. Additionally, 2-carbonylthiophene moiety occupied the linker region of HDAC. Furthermore, the 3-phenyl-5, 6, 7, 8-tetrahydroimidazo $[1,2$-a]pyrazine moiety occupied the cap pocket of the receptor (Table 2; Figures 4 and 5 ).

Compound 1 occupied the active cavity of HDAC showing a binding energy of $-18.70 \mathrm{kcal} / \mathrm{mol}$. Acetic acid moiety occupied the zinc binding and the carboxyl group of acetic acid moiety acted as zinc coordinator forming one hydrogen bond with Gly167. Also, it formed an electrostatic interaction with the $\mathrm{Zn}$ atom of the cocrystallized ligand. Additionally, (S)-2-methyltetrahydrofuran moiety occupied the linker region of HDAC. Furthermore, the $(4 \mathrm{aR}$, 8aS)-1,1,4a-trimethyl-1, 2, 3, 4, 4a, 5, 8, 8a-octahydronaphthalene moiety occupied the cap pocket of the receptor (Figures 4 and 6).

\section{Conclusions}

Bioassay-guided isolation of methanolic extract of Cousinia alata led to the identification of grindelic acid (1). Grindelic acid exhibited promising cytotoxic activities against Artemia salina nauplii and antibacterial activities against $S$. aureus, B. cereus, and S. enteritidis.

The pharmacofeatures and molecular docking studies revealed that (1) has a binding mode of interaction similar to that of the cocrystallized ligand and occupied the active cavity of HDAC enzyme with a binding energy of -18.70 , which may explain its noticeable biological activities. Additionally, six flavonoids (2-7) have been isolated from the chloroform extract of Cousinia alata. All compounds were isolated for the first time from Cousinia species.

\section{Data Availability}

The supporting data (NMR of compounds 1-7) are openly available.

\section{Conflicts of Interest}

The authors declare no conflicts of interest.

\section{Acknowledgments}

The authors thank Professor Wim De Borggraeve (KU Leuven, Belgium) for helping in NMR analysis and Zhanar Iskakova (the Kazakh University of Technology and Business) for his kind support and help. This research has been funded by the Science Committee of the Ministry of Education and Science of the Republic of Kazakhstan (Grant no. AP08051842).

\section{Supplementary Materials}

MS and NMR of the isolated compounds. (Supplementary Materials)

\section{References}

[1] R. L. Siegel, K. D. Miller, and A. Jemal, "Cancer statistics CA," A Cancer Journal for Clinicians, vol. 70, no. 1, p. 7, 2020.

[2] J. C. Bailar and H. L. Gornik, "Cancer undefeated," New England Journal of Medicine, vol. 336, no. 22, p. 1569, 1997.

[3] WHO. Cancer. 2020 [cited 2020 12-26];

[4] M. Frieri, K. Kumar, and A. Boutin, "Antibiotic resistance," Journal of Infection and Public Health, vol. 10, no. 4, p. 369, 2017. 
[5] M. H. Sharaf, G. M. El-Sherbiny, S. A. Moghannem et al., "New combination approaches to combat methicillin," Scientific Reports, vol. 11, p. 1, 2021.

[6] A. M. Metwaly, Z. Lianlian, H. Luqi, and D. Deqiang, "Black ginseng and its saponins: preparation, phytochemistry and pharmacological effects," Molecules, vol. 24, no. 10, p. 1856, 2019.

[7] Y.-M. Wang, X.-K. Ran, M. Riaz et al., "Chemical constituents of stems and leaves of tagetespatula L. And its fingerprint," Molecules, vol. 24, no. 21, p. 3911, 2019.

[8] S. V. Sperstad, T. Haug, H.-M. Blencke, O. B. Styrvold, C. Li, and K. Stensvåg, "Antimicrobial peptides from marine invertebrates: challenges and perspectives in marine antimicrobial peptide discovery," Biotechnology Advances, vol. 29, no. 5, p. 519, 2011.

[9] A. El-Demerdash, A. M. Metwaly, A. Hassan et al., "Comprehensive virtual screening of the antiviral potentialities of marine polycyclic guanidine alkaloids against SARS-CoV-2 (COVID-19)," Biomolecules, vol. 11, no. 3, p. 460, 2021.

[10] A. M. Metwaly, A. S. Wanas, M. M. Radwan, S. A. Ross, and M. A. ElSohly, "New $\alpha$-Pyrone derivatives from the endophytic fungus Embellisia sp," Medicinal Chemistry Research, vol. 26, no. 8, p. 1796, 2017.

[11] A. Metwaly, H. Kadry, A. El-Hela, A. Elsalam, and S. Ross, "New and antioxicant secondary metabolites from the endophytic fungus Nigrospora sphaerica," Planta Medica, vol. 80 , no. 10, p. PC11, 2014.

[12] A. Metwaly, "Comparative biological evaluation of four endophytic fungi isolated from nigella sativa seeds," Journal of Pharmaceutical Sciences, vol. 59, no. 1, p. 123, 2019.

[13] B. B. Basnet, L. Liu, B. Chen et al., "Four new cytotoxic arborinane-type triterpenes from the endolichenic fungus myrothecium inundatum," Planta Medica, vol. 85, no. 9, p. 701, 2019.

[14] B. B. Basnet, B. Chen, Y. M. Suleimen et al., "Cytotoxic secondary metabolites from the endolichenic fungus hypoxylon fuscum," Planta Medica, vol. 85, no. 13, p. 1088, 2019.

[15] M. M. Ghoneim, W. M. Afifi, M. Ibrahim et al., "Biological evaluation and molecular docking," Pharmacognosy Magazine, vol. 15, p. 232, 2019

[16] L. Liu, S. Luo, M. Yu et al., "Chemical constituents of tagetes patula and their," Natural Product Communications, vol. 15, 2020.

[17] E. M. Suleimen, R. I. Dzhalmakhanbetova, and M. Y. Ishmuratova, "Flavonoids from artemisia santolinifolia," Chemistry of Natural Compounds, vol. 50, no. 5, p. 918, 2014.

[18] A. M. Yassin, N. M. El-Deeb, A. M. Metwaly, G. F. El Fawal, M. M. Radwan, and E. E. Hafez, "Induction of apoptosis in human cancer cells through extrinsic and intrinsic pathways by balanites aegyptiaca furostanol saponins and saponincoated SilverNanoparticles," Applied Biochemistry and Biotechnology, vol. 182, no. 4, p. 1675, 2017.

[19] A. M. Metwaly, F. R. Fronczek, G. Ma et al., "Antileukemic $\alpha$-pyrone derivatives from the endophytic fungus Alternaria phragmospora," Tetrahedron Letters, vol. 55, no. 24, p. 3478 , 2014.

[20] A. M. Metwaly, H. A. Kadry, A. A. El-Hela et al., "Nigrosphaerin A a new isochromene derivative from the endophytic fungus Nigrospora sphaerica," Phytochemistry Letters, vol. 7, p. 1, 2014.

[21] A. M. Metwaly, M. M. Ghoneim, and A. Musa, "Biological evaluation and molecular docking," Derpharmachemica, vol. 7, p. 322, 2015.
[22] S. E. Sajjadi, M. Ghanadian, and M. Haghighi, Advanced Biomedical Research, vol. 6, 2017.

[23] J. Lindley, "A natural system of botany, or, a systematic view of the organization, natural affinities, and geographical distribution, of the whole vegetable kingdom: together with the uses of the most important species in medicine, the arts, and rural or domestic economy," 1836.

[24] Ulukuş, D. and Tugay, O.; 2020, 52, 297.

[25] Flora of China. 2007. p. 149.

[26] Flora of Kazakhstan. Alma-ata. 2007. p. 318.

[27] E. M. Suleimen, A. S. Zhanzhaksina, G. G. Sisengalieva, Z. B. Iskakova, and M. Y. Ishmuratova, "Constituent composition and biological activity of essential oil from Cousinia alata," Chemistry of Natural Compounds, vol. 54, no. 3, p. 595, 2018.

[28] L. Pasayeva, O. Üstün, E. Demirpolat, G. Karatoprak, O. Tugay, and M. Kosar, "Bioactivity-guided isolation of cytotoxic and antioxidant phytochemicals from four Cousinia species from stenocephala bunge section," Pharmacognosy Magazine, vol. 15, no. 65, p. 682, 2019.

[29] L. Paşayeva, O. Üstün, E. Demirpolat, G. Ş. Karatoprak, O. Tugay, and M. Koşar, Multidisciplinary Digital Publishing Institute Proceedings, vol. 1, p. 1001, 2017.

[30] S. E. Sajjadi, M. Ghanadian, M. Haghighi, and L. Mouhebat, "Faculty of microsystem electronics and photonics," Journal of HerbMed Pharmacology, vol. 4, p. 15, 2015.

[31] M. S. Amiri, H. A. Hosseini, and P. Rajai, "Study of antibacterial potentiality of leaf and fruit extracts," Asian Journal of Medical and Pharmaceutical Researches, vol. 4, p. 156, 2014.

[32] S. Bahraminejad, S. Abbasi, and R. Amiri, "The effect of some medicinal and ornamental plant extracts," Journal of Crop Protection, vol. 4, p. 189, 2015.

[33] A. Duran, N. Uslu, B. Dogan, M. Ozcan, and M. Çelik, "HPLC analysis and antioxidant, antibacterial and cytotoxicity," Journal of Agroalimentary Processes and Technologies, vol. 21, p. 136, 2015.

[34] K. Turdumambetov, N. V. Plekhanova, and D. A. Rakhimov, "Polysaccharides of subspecies of Cousinia," Chemistry of Natural Compounds, vol. 24, no. 5, p. 641, 1988.

[35] K. T. Turdumambetov, D. Azhibaeva, R. A. Goncharova, and E. E. Ernazarova, "Carbohydrate Cousinia angreni jus (Asteraceae), establishing the structure of their glu-cofructans," Chemistry of Plant Raw Material, vol. 1, no. 1, p. 41, 2020.

[36] J. A. Marco, J. F. Sanz, R. Albiach, A. Rustaiyan, and Z. Habibi, "Bisabolene derivatives and sesquiterpene lactones from Cousinia species," Phytochemistry, vol. 32, no. 2, p. 395, 1993.

[37] M. Iranshahy, Z. Tayarani-Najaran, J. Kasaian et al., "Highly oxygenated sesquiterpene lactones fromCousinia aitchisoniiand their cytotoxic properties: rhaserolide induces apoptosis in human T lymphocyte (jurkat) cells via the activation of c-Junn-terminal kinase phosphorylation," Phytotherapy Research, vol. 30, no. 2, p. 222, 2016.

[38] E. Verdin, Histone Deacetylases: Transcriptional Regulation and Other Cellular Functions, Springer Science \& Business Media, Berlin, Germany, 2007.

[39] A. Iglesias-Linares, R. M. Yañez-Vico, and M. A. GonzálezMoles, "Potential role of HDAC inhibitors in cancer therapy: insights into oral squamous cell carcinoma," Oral Oncology, vol. 46 , no. 5 , p. $323,2010$.

[40] G. Milazzo, D. Mercatelli, G. Di Muzio et al., "Histone deacetylases (HDACs): evolution, specificity, role in transcriptional complexes, and pharmacological actionability," Genes, vol. 11, no. 5, p. 556, 2020. 
[41] X. Wang, X. Tang, Z. Zhou, and Q. Huang, "Histone deacetylase 6 inhibitor enhances resistance to Mycobacterium tuberculosis infection through innate and adaptive immunity in mice," Pathogens and Disease, vol. 76, no. 6, Article ID fty064, 2018.

[42] G. Kasotakis, M. Galvan, E. King et al., "Valproic acid mitigates the inflammatory response and prevents acute respiratory distress syndrome in a murine model of Escherichia coli pneumonia at the expense of bacterial clearance," Journal of Trauma and Acute Care Surgery, vol. 82, no. 4, p. 758, 2017.

[43] K. B. Lagosz and A. M. Grabiec, "Targeting histone deacetylases for bacterial infections," in Histone Modifications in TherapyElsevier, Amsterdam, Netherlands, 2020.

[44] S. K. Panda, L. Padhi, P. Leyssen, M. Liu, J. Neyts, and W. Luyten, "Antimicrobial, anthelmintic, and antiviral activity," Frontiers in Pharmacology, vol. 8, p. 658, 2017.

[45] N. Martins, S. Petropoulos, and I. C. F. R. Ferreira, "Chemical composition and bioactive compounds of garlic (Allium sativum L.) as affected by pre- and post-harvest conditions: a review," Food Chemistry, vol. 211, p. 41, 2016.

[46] K. M. El-Gamal, A. M. El-Morsy, A. M. Saad, I. H. Eissa, and M. Alswah, "Synthesis, docking, QSAR, ADMET and antimicrobial evaluation of new quinoline-3-carbonitrile derivatives as potential DNA-gyrase inhibitors," Journal of Molecular Structure, vol. 1166, p. 15, 2018.

[47] I. H. Eissa, M. K. Ibrahim, A. M. Metwaly et al., "Screening of some sulfonamide and sulfonylurea derivatives as Anti-Alzheimer's Agents Targeting BACE1 and PPAR $\gamma$," Bioorganic Chemistry, vol. 2020, Article ID 1631243, 2020.

[48] A. A. Nasser, I. H. Eissa, M. R. Oun et al., "Discovery of new pyrimidine-5-carbonitrile derivatives as anticancer agents targeting EGFRWT and EGFRT790M," Organic \& Biomolecular Chemistry, vol. 18, no. 38, p. 7608, 2020.

[49] M. K. Ibrahim, I. H. Eissa, M. S. Alesawy, A. M. Metwaly, M. M. Radwan, and M. A. ElSohly, "Design, synthesis, molecular modeling and anti-hyperglycemic evaluation of quinazolin-4(3H)-one derivatives as potential PPAR $\gamma$ and SUR agonists," Bioorganic \& Medicinal Chemistry, vol. 25, no. 17, p. 4723, 2017.

[50] S. A. Elmetwally, K. F. Saied, I. H. Eissa, and E. B. Elkaeed, "Design, synthesis and anticancer evaluation of thieno[2,3-d] pyrimidine derivatives as dual EGFR/HER2 inhibitors and apoptosis inducers," Bioorganic Chemistry, vol. 88, Article ID 102944, 2019.

[51] H. A. Mahdy, M. K. Ibrahim, A. M. Metwaly et al., "Design, synthesis, molecular modeling, in vivo studies and anticancer evaluation of quinazolin- $4(3 \mathrm{H})$-one derivatives as potential VEGFR-2 inhibitors and apoptosis inducers," Bioorganic Chemistry, vol. 94, Article ID 103422, 2020.

[52] M. A. El-Zahabi, E. R. Elbendary, F. H. Bamanie, M. F. Radwan, S. A. Ghareib, and I. H. Eissa, "Design, synthesis, molecular modeling and anti-hyperglycemic evaluation of phthalimide-sulfonylurea hybrids as PPAR $\gamma$ and SUR agonists," Bioorganic Chemistry, vol. 91, Article ID 103115, 2019.

[53] A. M. El-Naggar, I. H. Eissa, A. Belal, and A. A. El-Sayed, "Design, eco-friendly synthesis, molecular modeling and anticancer evaluation of thiazol-5(4H)-ones as potential tubulin polymerization inhibitors targeting the colchicine binding site," RSC Advances, vol. 10, no. 5, p. 2791, 2020.

[54] X. E. Hernandez, A. A. Orden, O. S. Giordano, and M. Kurina, "Effects of elicitor and copper sulfate on grindelic acid," Electronic Journal of Biotechnology, vol. 8, 2005.
[55] G. Citoğlu, B. Sever, S. Antus, E. Baitz-Gacs, and N. Altanlar, "Antifungal flavonoids from ballota glandulosissima," Pharmaceutical Biology, vol. 41, p. 483, 2003.

[56] M. B. Barreto, C. L. Gomes, J. V. B. D. Freitas et al., "Flavonoides e terpenoides de Croton muscicarpa (Euphorbiaceae)," Química Nova, vol. 36, no. 5, p. 675, 2013.

[57] H. Dong, Y.-L. Gou, S.-G. Cao et al., "Eicosenones and methylated flavonols from Amomumkoenigii," Phytochemistry, vol. 50, no. 5, p. 899, 1999.

[58] Y. Wang, M. Hamburger, J. Gueho, and K. Hostettmann, "Antimicrobial flavonoids from Psiadia trinervia and their methylated and acetylated derivatives," Phytochemistry, vol. 28, no. 9, p. 2323, 1989.

[59] Y.-J. Li, Y. Guo, Q. Yang et al., "Flavonoids casticin and chrysosplenol D from Artemisia annua L. inhibit inflammation in vitro and in vivo," Toxicology and Applied Pharmacology, vol. 286, no. 3, p. 151, 2015.

[60] F. Torres, J. Quintana, and F. Estévez, “5,7,3'-trihydroxy-3,4' dimethoxyflavone inhibits the tubulin polymerization and activates the sphingomyelin pathway," Molecular Carcinogenesis, vol. 50, no. 2, p. 113, 2011.

[61] P. W. Atadja, "HDAC inhibitors and cancer therapy," in Epigenetics and Diseasep. 175, Springer, Berlin, Germany, 2011.

[62] Y. Li, Y. Wang, N. Xie et al., "Design, synthesis and antiproliferative activities of novel benzamides derivatives as HDAC inhibitors," European Journal of Medicinal Chemistry, vol. 100, p. 270, 2015.

[63] R. Sangwan, R. Rajan, and P. K. Mandal, "HDAC as onco target: reviewing the synthetic approaches with SAR study of their inhibitors," European Journal of Medicinal Chemistry, vol. 158, p. 620, 2018. 